\title{
An Impulse Dynamic Model for Computer Worms
}

\author{
Chunming Zhang, Yun Zhao, and Yingjiang Wu \\ School of Information Engineering, Guangdong Medical College, Dongguan 523808, China \\ Correspondence should be addressed to Chunming Zhang; chunfei2002@163.com
}

Received 3 May 2013; Accepted 2 June 2013

Academic Editor: Luca Guerrini

Copyright (C) 2013 Chunming Zhang et al. This is an open access article distributed under the Creative Commons Attribution License, which permits unrestricted use, distribution, and reproduction in any medium, provided the original work is properly cited.

A worm spread model concerning impulsive control strategy is proposed and analyzed. We prove that there exists a globally attractive virus-free periodic solution when the vaccination rate is larger than $\theta_{1}$. Moreover, we show that the system is uniformly persistent if the vaccination rate is less than $\theta_{1}$. Some numerical simulations are also given to illustrate our main results.

\section{Introduction}

Computer virus is a kind of computer program that can replicate itself and spread from one computer to others including viruses, worms, and trojan horses. Worms use system vulnerability to search and attack computers. As hardware and software technologies develop and computer networks become an essential tool for daily life, worms start to be a major threat. In June 2010, the Belarusian security firm Virus Block Ada discovered deadly Stuxnet worm. The Stuxnet worm is the first known example of a cyber-weapon that is designed not just to steal and manipulate data but to attack a processing system and cause physical damage. The Stuxnet worm is the first cyber-attack of its kind and has infected thousands of computer systems worldwide.

Consequently, the trial on better understanding the worm propagation dynamics is an important matter for improving the safety and reliability in computer systems and networks. Similar to the biological viruses, there are two ways to study this problem: microscopic and macroscopic. Following a macroscopic approach, since $[1,2]$ took the first step towards modeling the spread behavior of worms, much effort has been done in the area of developing a mathematical model for the worms propagation [3-13]. These models provide a reasonable qualitative understanding of the conditions under which viruses spread much faster than others and why.

In [7], the authors investigated a differential SEIR model by making the following assumptions (Figure 1).

A population size $N(t)$, that is, the total nodes at any time $t$ in the computer network, is partitioned into subclasses of nodes which are susceptible, exposed (infected but not yet infectious), infectious, and recovered with sizes denoted by $S(t), E(t), I(t)$, and $R(t)$, respectively.

One has

$$
\begin{gathered}
N(t)=S(t)+E(t)+I(t)+R(t), \\
S^{\prime}(t)=b-\lambda I S-p b E-q b I-d S+\zeta R, \\
E^{\prime}(t)=\lambda I S+p b E+q b I-\varepsilon E-d E, \\
I^{\prime}(t)=\varepsilon E-\gamma I-d I-\eta I, \\
R^{\prime}(t)=\gamma I-\zeta R-d R,
\end{gathered}
$$

where $b, d$, and $\lambda$ are positive constants and $\varepsilon, \eta, \gamma, \zeta$ are nonnegative constants. The constant $b$ is the recruitment rate of susceptible nodes to the computer network, $d$ is the per capita natural mortality rate (i.e., the crashing of nodes due to the reason other than the attack of worms), $\varepsilon$ is the rate constant for nodes leaving the exposed class $E$ for infective class $I, \gamma$ is the rate constant for nodes leaving the infective class $I$ for recovered class $R, \eta$ is the disease related death rate (i.e., crashing of nodes due to the attack of worms) in the class $I$, and $\zeta$ is the rate constant for nodes becoming susceptible again after recovering.

In the SEIRS model, the flow is from class $S$ to class $E$, class $E$ to class $I$, class $I$ to class $R$, and again class $R$ to class $S$. For the vertical transformation, we assume that a fraction $p$ and a fraction $q$ of the new nodes from the exposed and the infectious classes, respectively, are introduced into 


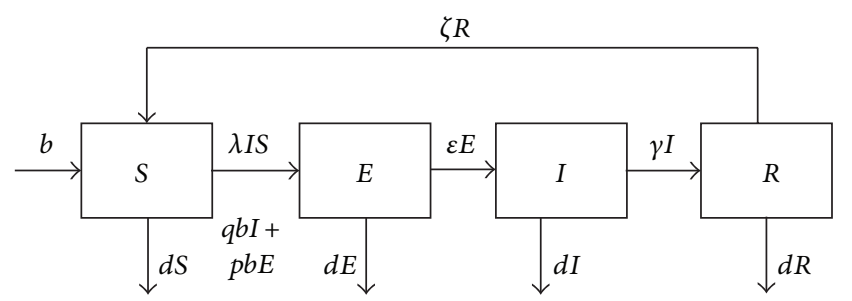

FIGURE 1: Original model.

the exposed class $E$. Consequently, the birth flux into the exposed class is given by $p b E+q b I$, and the birth flux into the susceptible class is given by $b-p b E-q b I$.

As we know, antivirus software is a kind of computer program which can detect and eliminate known worm. There are two common methods to detect worms: using a list of worm signature definition and using a heuristic algorithm to find worm based on common behaviors. It has been observed that it does not always work in detecting a novel worm by using the heuristic algorithm. On the other hand, obviously, it is impossible for antivirus software to find a new worm signature definition on the dated list. So, to keep the antivirus software in high efficiency, it is important to ensure that it is updated. Based on the previous facts, we propose an impulsive system to model the process of periodic installing or updating antivirus software on susceptible computers at fixed time for controlling the spread of worm.

Based on the previous facts, we propose the following assumptions:

(H1) the antivirus software is installed or updated at time $t=k \tau(k \in N)$, where $\tau$ is the period of the impulsive effect;

(H2) $S$ computers are successfully vaccinated from $S$ class to $R$ class with rate $\theta(0<\theta<1)$.

According to the previous assumptions $(H 1)-(H 2)$ and for the reason of simplicity, we propose the following model (Figure 2):

$$
\begin{aligned}
& S^{\prime}(t)=b-\lambda I S-p b E-q b I-d S+\zeta R, \\
& E^{\prime}(t)=\lambda I S+p b E+q b I-\varepsilon E-d E, \quad t \neq k \tau, k \in Z^{+}, \\
& I^{\prime}(t)=\varepsilon E-\gamma I-d I-\eta I, \\
& R^{\prime}(t)=\gamma I-\zeta R-d R, \\
& S\left(t^{+}\right)=(1-\theta) S(t), \\
& E\left(t^{+}\right)=E(t), \quad t=k \tau, k \in Z^{+} . \\
& I\left(t^{+}\right)=I(t), \quad \\
& R\left(t^{+}\right)=R(t)+\theta S(t),
\end{aligned}
$$

The total population size $N(t)$ can be determined by $N(t)=$ $S(t)+E(t)+I(t)+R(t)$ to form the differential equation

$$
N^{\prime}(t)=b-d N(t)-\eta I(t)
$$

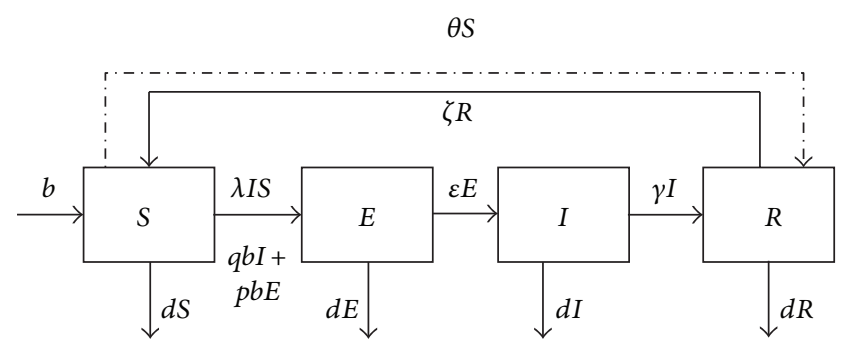

FIGURE 2: Impulse model.

which is derived by adding the equations in system (1). Thus the total population size $N$ may vary in time. From (2), we have

$$
b-(d+\eta) N(t) \leq N^{\prime}(t) \leq b-d N(t) .
$$

It follows that

$$
\frac{b}{(d+\eta)} \leq \lim _{x \rightarrow \infty} \inf N(t) \leq \lim _{x \rightarrow \infty} \sup N(t) \leq \frac{b}{d} .
$$

The system (2) can be reduced to the equivalent system

$$
\begin{aligned}
& S^{\prime}(t)=b-\lambda I S-p b E-q b I-d S \\
& +\zeta(N-S-E-I), \\
& E^{\prime}(t)=\lambda I S+p b E+q b I \\
& -\varepsilon E-d E, \\
& I^{\prime}(t)=\varepsilon E-\gamma I-d I-\eta I, \\
& N^{\prime}(t)=b-d N(t)-\eta I(t), \\
& \quad S\left(t^{+}\right)=(1-\theta) S(t), \quad \\
& \quad E\left(t^{+}\right)=E(t), \\
& \quad I\left(t^{+}\right)=I(t), \\
& \quad N\left(t^{+}\right)=N(t) .
\end{aligned}
$$

The initial conditions for (6) are

$$
S\left(0^{+}\right)>0, \quad E\left(0^{+}\right)>0, \quad I\left(0^{+}\right)>0, \quad N\left(0^{+}\right)>0 .
$$

From physical considerations, we discuss system (6) in the closed set

$$
\Omega=\left\{(S, E, I, N) \in R_{+}^{4} \mid 0 \leq S+E+I \leq \frac{b}{d}, 0 \leq N \leq \frac{b}{d}\right\} .
$$

The organization of this paper is as follows. In Section 2, we establish sufficient condition for the local and global attractivity of virus-free periodic solution. The sufficient condition for the permanence of the model is obtained in Section 3. Some numerical simulations are performed in Section 4 . In the final section, a brief conclusion is given, and some future research directions are also pointed out. 


\section{Global Attractivity of Virus-Free Periodic Solution}

To prove our main results, we state three lemmas which will be essential to our proofs.

Lemma 1 (see [14]). Consider the following impulsive differential equations:

$$
\begin{gathered}
\dot{u}(t)=a-b u(t), \quad t \neq k \tau, \\
u\left(t^{+}\right)=(1-\theta) u(t), \quad t=k \tau,
\end{gathered}
$$

where $a>0, b>0$, and $0<\theta<1$. Then system (9) has $a$ unique positive periodic solution

$$
\bar{u}_{e}(t)=\frac{a}{b}+\left(u^{*}-\frac{a}{b}\right) e^{-b(t-k \tau)}, \quad k \tau<t \leq(k+1) \tau,
$$

which is globally asymptotically stable; there $u^{*}=a(1-\theta)(1-$ $\left.e^{-b \tau}\right) / b\left(1-(1-\theta) e^{-b \tau}\right)$.

If $I(t) \equiv 0$, we have the following limit systems:

$$
\begin{aligned}
S^{\prime}(t)= & b-\lambda I S-p b E-q b I \\
& -d S+\zeta(N-S-E), \quad t \neq k \tau, k \in Z^{+}, \\
E^{\prime}(t)= & p b E-\varepsilon E-d E, \quad \\
N^{\prime}(t)= & b-d N, \\
& S\left(t^{+}\right)=(1-\theta) S(t), \\
& E\left(t^{+}\right)=E(t), \quad t=k \tau, k \in Z^{+}, \\
& N\left(t^{+}\right)=N(t) .
\end{aligned}
$$

When $p b-d-\varepsilon<0$, there exists $t_{1}$ when $t>t_{1}, \lim _{t \rightarrow \infty} E(t)=$ 0 . From the third and sixth equations of system (11), we have $\lim _{t \rightarrow \infty} N(t)=b / d$. We have the following limit systems:

$$
\begin{gathered}
\frac{d S}{d t}=(1-p) b+\frac{v b}{\mu}-(\mu+v) S, \quad t \neq k T, k \in Z^{+}, \\
S\left(t^{+}\right)=(1-\theta) S(t), \quad t=k T .
\end{gathered}
$$

According to Lemma 1, we know that periodic solution of system (12) is of the form

$$
\begin{aligned}
& \bar{S}_{e}(t)= \frac{(\mu(1-p)+v) b}{\mu(\mu+v)} \\
&+\left(S^{*}-\frac{(\mu(1-p)+v) b}{\mu(\mu+v)}\right) e^{-(\mu+v)(t-k \tau),} \\
& k \tau<t \leq(k+1) \tau,
\end{aligned}
$$

and it is globally asymptotically stable, where $S^{*}=((1-p) b+$ $(v b / \mu))(1-\theta)\left(e^{(\mu+v) \tau}-1\right) /(\mu+v)\left(e^{(\mu+v) \tau}-1+\theta\right)$.

Theorem 2. Let $(S(t), E(t), I(t), N(t))$ be any solution of system (6) with initial values $S\left(0^{+}\right)>0, E\left(0^{+}\right)>0, I\left(0^{+}\right)>0$, and $N\left(0^{+}\right)>0$; then $\left(\bar{S}_{e}(t), 0,0, b / d\right)$ is locally asymptotically stable, provided that $p b-d-\varepsilon<0$ and $R_{0}<1$, where

$$
\begin{aligned}
R_{0}= & \frac{1}{(d+\varepsilon-p b)(\gamma+d+\eta) \tau-\varepsilon q b \tau} \\
& \times \varepsilon \lambda \frac{b}{d}\left[\tau+\frac{\theta\left(1-e^{(\zeta+d) \tau}\right)}{(\zeta+d)\left(e^{(\zeta+d) \tau}-1+\theta\right)}\right] .
\end{aligned}
$$

Proof. The local stability of virus-free periodic solution may be determined by considering the behaviors of a small amplitude perturbation of the solution. Define $w(t)=S(t)-$ $\bar{S}_{e}(t), x(t)=E(t), y(t)=I(t)$, and $z(t)=N(t)-b / d$, and then the linearized system of system (6) reads as

$$
\begin{aligned}
& \begin{aligned}
w^{\prime}(t)= & -(d+\zeta) w(t)-(p b+\zeta) x(t) \\
& -\left(\lambda \bar{S}_{e}(t)+\zeta+q b\right) y(t)+\zeta z(t),
\end{aligned} \\
& \begin{aligned}
x^{\prime}(t)= & (p b-d-\varepsilon) x(t) \\
& +\left(q b+\lambda \bar{S}_{e}(t)\right) y(t),
\end{aligned} \quad t \neq k \tau, k \in Z^{+}, \\
& y^{\prime}(t)= \varepsilon x(t)-(r+d+\eta) y(t), \\
& z^{\prime}(t)=-\eta y(t)-d z(t), t=k \tau, k \in Z^{+}, \\
& w\left(t^{+}\right)=w(t), \\
& x\left(t^{+}\right)=x(t), \\
& y\left(t^{+}\right)=y(t), \\
& z\left(t^{+}\right)=z(t) .
\end{aligned}
$$

Let $\Phi(t)$ be the fundamental solution matrix of system (15), and then $\Phi(t)$ must satisfy

$$
\begin{aligned}
& \frac{d \Phi(t)}{d t} \\
& \quad=\left(\begin{array}{cccc}
-d-\zeta & -p b-\zeta & -\lambda \bar{S}_{e}(t)-\zeta-q b & \zeta \\
0 & p b-d-\varepsilon & q b+\lambda \bar{S}_{e}(t) & 0 \\
0 & \varepsilon & -r-d-\eta & 0 \\
0 & 0 & -\eta & -d
\end{array}\right) \Phi(t) \\
& \doteq A \Phi(t),
\end{aligned}
$$

and $\Phi(0)=I$, the identity matrix. We can easily see that two eigenvalues of the matrix $A$ are $-d-\zeta$ and $-d$, and the other two eigenvalues are determined by the $2 \times 2$ matrix $B=$ $\left(\begin{array}{cc}p b-d-\varepsilon & q b+\lambda \bar{S}_{e}(t) \\ \varepsilon & -r-d-\eta\end{array}\right)$. Denote the eigenvalues of $B$ as $\lambda_{1}, \lambda_{2}$, and then as $p b-d-\varepsilon<0$, we have $\lambda_{1}+\lambda_{2}=p b-d-\varepsilon-r-d-\eta<0$,

$$
\lambda_{1} \lambda_{2}=(p b-d-\varepsilon)(-r-d-\eta)-\varepsilon\left(q b+\lambda \bar{S}_{e}(t)\right) .
$$


Therefore, by the Floquet theorem [15], $\left(\bar{S}_{e}(t), 0,0, b / d\right)$ is locally asymptotically stable, provided that

$$
\begin{aligned}
G= & \int_{0}^{\tau}\left[(p b-d-\varepsilon)(-\gamma-d-\eta)-\varepsilon\left(q b+\lambda \bar{S}_{e}(t)\right)\right] d t \\
= & (p b-d-\varepsilon)(-\gamma-d-\eta) \tau-\varepsilon q b \tau \\
& -\varepsilon \lambda \frac{b}{d}\left[\tau+\frac{\theta\left(1-e^{(\zeta+d) \tau}\right)}{(\zeta+d)\left(e^{(\zeta+d) \tau}-1+\theta\right)}\right]>0 .
\end{aligned}
$$

When $R_{0}=(1 /((d+\varepsilon-p b)(\gamma+d+\eta) \tau-\varepsilon q b \tau)) \varepsilon \lambda(b / d)[\tau+\theta(1-$ $\left.\left.e^{(\zeta+d) \tau}\right) /(\zeta+d)\left(e^{(\zeta+d) \tau}-1+\theta\right)\right] \leq 1$, the previous inequality is satisfied for $p b-d-\varepsilon<0$.

The proof is complete.

Theorem 3. If $R_{1} \leq 1$, and $p b-d-\varepsilon<0$ then $\left(\bar{S}_{e}(t), 0,0, b / d\right)$ is globally asymptotically stable for system (11), where

$$
\begin{aligned}
R_{1}= & \frac{1}{(d+\varepsilon-p b)(\gamma+d+\eta) \tau-\varepsilon q b \tau} \varepsilon \lambda \frac{b \tau}{d} \\
& \times\left[1-\frac{\theta}{\left(e^{(\zeta+d) \tau}-1+\theta\right)}\right] .
\end{aligned}
$$

Proof. Because $e^{x}-1>x$, for $x>0$, we have

$$
R_{0}<R_{1}
$$

By Theorem 2 , we know that $\left(\bar{S}_{e}(t), 0,0, b / d\right)$ is locally asymptotically stable. In the following, we will prove the global attraction of $\left(\bar{S}_{e}(t), 0,0, b / d\right)$.

Let

$$
L=\varepsilon E-(p b-\varepsilon-d) I .
$$

Then

$$
\begin{gathered}
L^{\prime}=\varepsilon E^{\prime}-(p b-\varepsilon-d) I^{\prime} \\
L^{\prime}=[\varepsilon \lambda S+\varepsilon q b+(p b+\varepsilon-d)(r+d+\eta)] I .
\end{gathered}
$$

Therefore, $\left(\bar{S}_{e}(t), 0,0, b / d\right)$ is globally asymptotically stable, provided that

$$
\begin{aligned}
L^{\prime}= & \int_{0}^{\tau}[\varepsilon \lambda S+\varepsilon q b+(p b+\varepsilon-d)(r+d+\eta)] I d t<0, \\
L^{\prime}= & \int_{0}^{\tau}[\varepsilon \lambda S+\varepsilon q b+(p b+\varepsilon-d)(r+d+\eta)] d t \\
< & {[\varepsilon q b+(p b+\varepsilon-d)(r+d+\eta)] \tau } \\
& +\varepsilon \lambda \frac{b}{d}\left(1-\frac{\theta}{e^{(d+\zeta) \tau}-1+\theta}\right) \tau<0 .
\end{aligned}
$$

When $R_{1}=(1 /((d+\varepsilon-p b)(\gamma+d+\eta) \tau-\varepsilon q b \tau)) \varepsilon \lambda(b \tau / d)[1-$ $\left.\theta /\left(e^{(\zeta+d) \tau}-1+\theta\right)\right] \leq 1$, the previous inequality is satisfied.

The proof is complete.
Corollary 4. The virus-free periodic solution $\left(\bar{S}_{e}(t), 0,0, b / d\right)$ of system (6) is globally attractive, if $\theta>\theta_{1}$, where $\theta_{1}=1-$ $e^{(\zeta+d) \tau}+\varepsilon b \lambda\left(e^{(\zeta+d) \tau}-1\right) / d[(d+\varepsilon-p b)(r+d+\eta)-\varepsilon q b]$.

Theorem 2 determines the global attractivity of (6) in $\Omega$ for the case $R_{0}<1$. Its realistic implication is that the infected computers vanish, so the worms are removed from the network. Corollary 4 implies that the computer virus will disappear if the vaccination rate is less than $\theta_{1}$.

\section{Permanence}

In this section, we say that the worm is local if the infected population persists above a certain positive level for sufficiently large time. The local of worm can be well captured and studied through the notion of permanence.

Definition 5. System (6) is said to be uniformly persistent if there is an $\varphi>0$ (independent of the initial data) such that every solution $(S(t), I(t), R(t), N(t))$ with initial conditions (8) of system (6) satisfies

$$
\begin{array}{ll}
\lim _{t \rightarrow \infty} \inf S(t) \geq \varphi, & \lim _{t \rightarrow \infty} \inf I(t) \geq \varphi, \\
\lim _{t \rightarrow \infty} \inf R(t) \geq \varphi, & \lim _{t \rightarrow \infty} \inf N(t) \geq \varphi .
\end{array}
$$

Theorem 6. Suppose that $R_{1}>1$ and $p b-d-\varepsilon<0$. Then there is a positive constant $m_{I}$ such that each positive solution $(S(t), E(t), I(t), R(t))$ of system (6) satisfies $I(t) \geq m_{I}$, for $t$ large enough.

Proof. Now, we will prove that there exist $m_{I}>0$ and a sufficiently large $t_{p}$ such that $I(t) \geq m_{I}$ holds for all $t>t_{p}$. Suppose that $I(t)<I^{*}$ for all $t>t_{0}$. From the forth equation of (6), we have

$$
\begin{gathered}
b-d N(t)>N^{\prime}(t)>b-d N(t)-\eta I^{*}, \\
\frac{b}{d}>N(t)>\frac{b-\eta I^{*}}{d} .
\end{gathered}
$$

From the second equation of (6), we have

$$
\begin{aligned}
E^{\prime}(t) & =\lambda I S+p b E+q b I-\varepsilon E-d E \\
& <\lambda I^{*} S+q b I^{*}+(p b-\varepsilon-d) E \\
& <\lambda I^{*} \frac{b}{d}+q b I^{*}+(p b-\varepsilon-d) E .
\end{aligned}
$$

As $p b-d-\varepsilon<0$, 
From the first equation of (6), we have

$$
\begin{aligned}
S^{\prime}(t)= & b-\lambda I S-p b E-q b I-d S+\zeta(N-S-E-I) \\
> & b+\zeta \frac{b-\eta I^{*}}{d}-q b I^{*}-\zeta I^{*} \\
& -(p b+\zeta) \frac{(q b+\lambda) b I^{*}}{d(\varepsilon+d-p b)}-\left(\lambda I^{*}+d+\zeta\right) S(t)
\end{aligned}
$$

Consider the following comparison system:

$$
\begin{gathered}
v^{\prime}(t)=b+\zeta \frac{b-\eta I^{*}}{d}-q b I^{*}-\zeta I^{*} \\
-(p b+\zeta) \frac{(q b+\lambda) b I^{*}}{d(\varepsilon+d-p b)}-\left(\lambda I^{*}+d+\zeta\right) v(t), \\
v\left(t^{+}\right)=(1-\theta) v(t), \quad t=k \tau, \\
v\left(0^{+}\right)=S\left(0^{+}\right) .
\end{gathered}
$$

let $A \doteq b+\zeta\left(\left(b-\eta I^{*}\right) / d\right)-q b I^{*}-\zeta I^{*}-(p b+\zeta)\left((q b+\lambda) b I^{*} / d(\varepsilon+\right.$ $d-p b)), B \doteq \lambda I^{*}+d+\zeta$.

By Lemma 1, we know that there exists $t_{1}>t_{0}$ such that

$$
\begin{gathered}
\bar{v}(t)=\frac{A}{B}+\left(v^{*}-\frac{A}{B}\right) e^{-B(t-k \tau)}, \quad k \tau<t \leq(k+1) \tau, \\
v^{*}=\frac{A(1-\theta)\left(1-e^{-B \tau}\right)}{B\left(1-(1-\theta) e^{-B \tau}\right)}, \\
S(t)>\bar{v}(t) \doteq S^{*}>0, \quad \text { for } t>t_{1} .
\end{gathered}
$$

From the second equation of (6), we have

$$
\begin{gathered}
E^{\prime}(t)=\lambda I S+p b E+q b I-\varepsilon E-d E \\
>\lambda I^{*} S^{*}+q b I^{*}-(\varepsilon+d-p b) E, \\
E(t)>\frac{\lambda I^{*} S^{*}+q b I^{*}}{\varepsilon+d-p b} .
\end{gathered}
$$

From the third equation of (6); we have

$$
\begin{aligned}
I^{\prime}(t) & =\varepsilon E-\gamma I-d I-\eta I \\
& >\varepsilon \frac{\lambda I^{*} S^{*}+q b I^{*}}{\varepsilon+d-p b}-(\gamma+d+\eta) I .
\end{aligned}
$$

Note that $R_{1}>1$, we have

$$
I(t)>\frac{\varepsilon}{\gamma+d+\eta} \frac{\lambda I^{*} S^{*}+q b I^{*}}{\varepsilon+d-p b}>I^{*} .
$$

This contradicts $I(t) \leq I^{*}$. Hence, we can claim that for any $t_{0}>0$, it is impossible that

$$
I(t)<I^{*} \quad \forall t \geq \mathrm{t}_{0} .
$$

By the claim, we are left to consider two cases. First, $I(t) \geq I^{*}$ for $t$ large enough. Second, $I(t)$ oscillates about $I^{*}$ for $t$ large enough. Obviously, there is nothing to prove for the first case. For the second case, we can choose $t_{2}>t_{1}$, and $\xi>0$ satisfy

$$
I\left(t_{2}\right)=I\left(t_{2}+\xi\right)=I^{*}, \quad \text { for } t_{2}<t<t_{2}+\xi
$$

$I(t)$ is uniformly continuous since the positive solutions of (6) are ultimately bounded, and $I(t)$ is not affected by impulses.

Therefore, it is certain that there exists a $\eta(0<\eta<\tau$, and $\eta$ is independent of the choice of $t_{2}$ ) such that

$$
I(t) \geq \frac{I^{*}}{2}, \quad \text { for } t_{2} \leq t \leq t_{2}+\eta
$$

In this case, we consider the following three possible cases in term of the sizes of $\eta$, $\xi$, and $\tau$.

Case 1. If $\xi \leq \eta<\tau$, then it is obvious that $I(t) \geq \mathrm{I}^{*} / 2$, for $t \in\left[t_{2}, t_{2}+\xi\right]$.

Case 2. If $\eta<\xi \leq \tau$, then from the second equation of system (6), we obtain $\dot{I}(t)>-(d+\gamma+\eta) I(t)$.

Since $I\left(t_{2}\right)=I^{*}$, it is obvious that $I(t)>I^{*} e^{-(d+\gamma+\eta) \tau} \doteq I^{* *}$, for $t \in\left[t_{2}, t_{2}+\xi\right]$.

Case 3. If $\eta<\tau \leq \xi$, it is easy to obtain that $I(t)>I^{* *}$ for $t \in\left[t_{2}, t_{2}+\tau\right]$. Then, proceeding exactly as the proof for the previous claim, we have that $I(t)>I^{* *}$ for $t \in\left[t_{2}+\tau, t_{2}+\xi\right]$.

Owing to the randomicity of $t_{2}$, we can obtain that there exists $m_{I} \doteq \min \left\{I^{*} / 2, I^{* *}\right\}$ such $I(t)>m_{I}$ holds for all $t>t_{p}$. The proof of Theorem 6 is completed.

Theorem 7. Suppose $R_{1}>1$. Then system (6) is permanent.

Proof. Let $(S(t), E(t), I(t), N(t))$ be any solution of system (6). First, from the first equation of system (6), we have

$$
S^{\prime}(t)>b-p b^{2}-q b^{2}-(d+\lambda b) S .
$$

Consider the following comparison system:

$$
\begin{gathered}
\dot{z}_{1}(t)=b-p b^{2}-q b^{2}-(d+\lambda b) z_{1}(t), \quad t \neq k \tau, \\
z_{1}\left(t^{+}\right)=(1-\theta) z_{1}(t), \quad t=k \tau .
\end{gathered}
$$

By Lemma 1, we know that for any sufficiently small $\varepsilon>0$, there exists a $t_{1}\left(t_{1}\right.$ is sufficiently large) such that

$$
\begin{aligned}
& S(t) \geq z_{1}(t)>z_{1}^{*}(t)-\varepsilon \\
& \geq \frac{(1-p b-q b) b\left(e^{(d+\lambda b) \tau}-1\right)}{(d+\lambda b)\left(e^{(d+\lambda b) \tau}-1+\theta\right)}-\varepsilon \doteq m_{S}>0, \\
& k \tau<t \leq(k+1) \tau .
\end{aligned}
$$

From (31), we have

$$
E(t)>\frac{\lambda m_{I} m_{S}+q b m_{I}}{\varepsilon+d-p b}-\varepsilon \doteq m_{E} .
$$




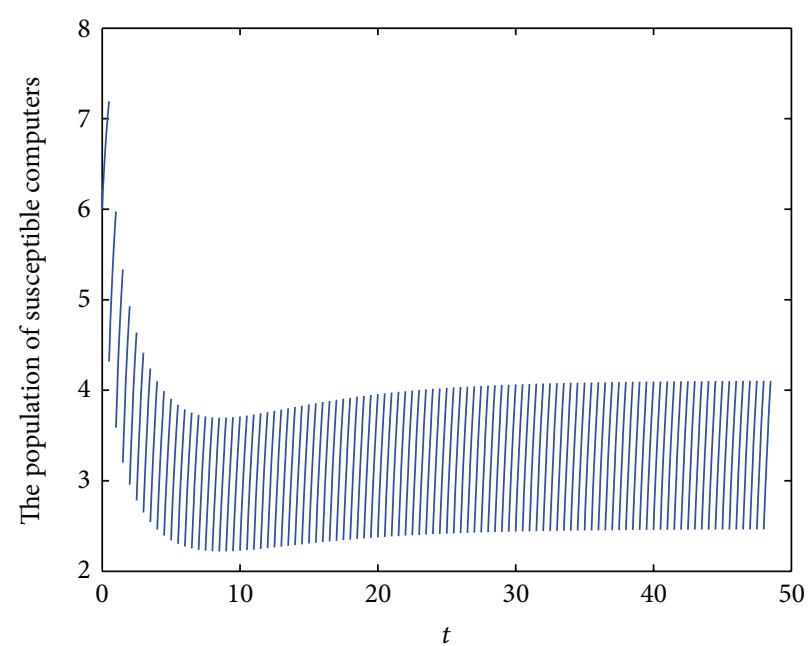

(a)

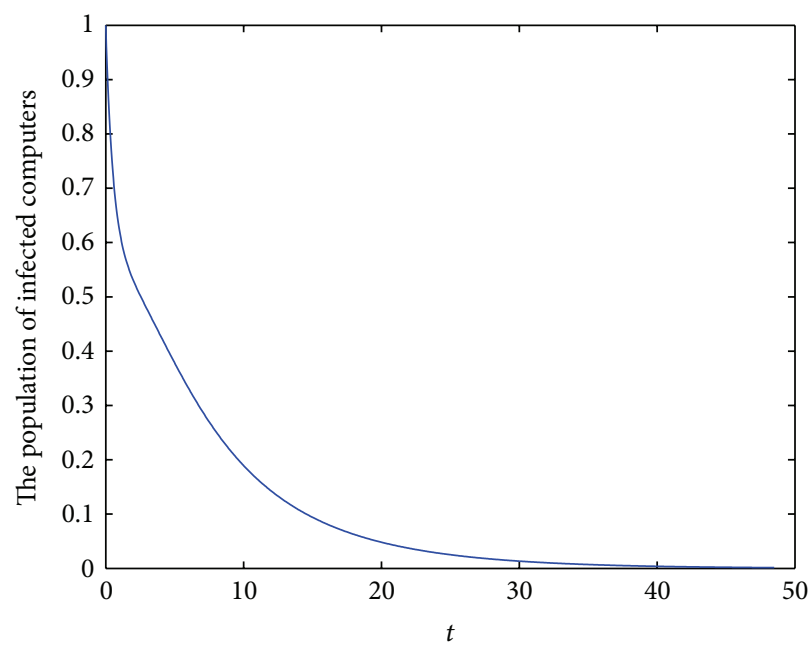

(c)

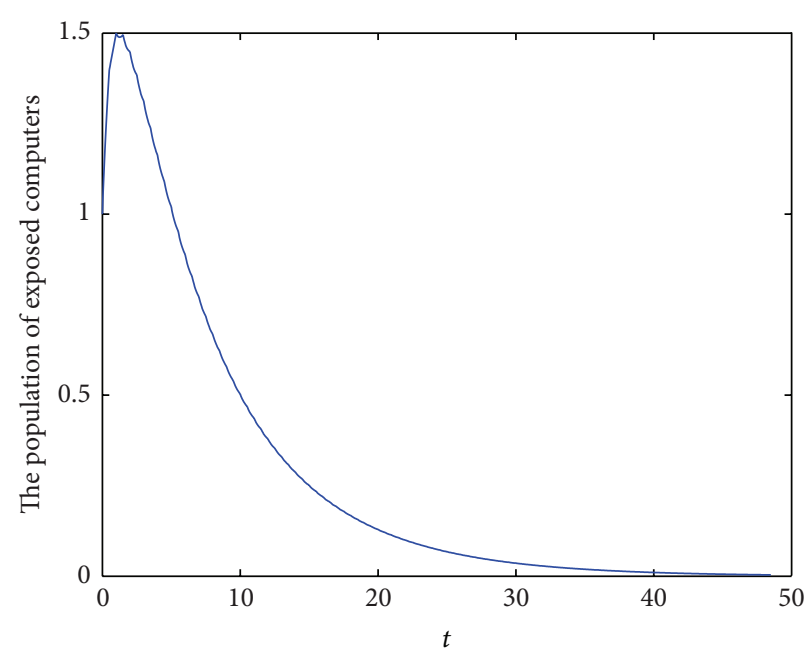

(b)

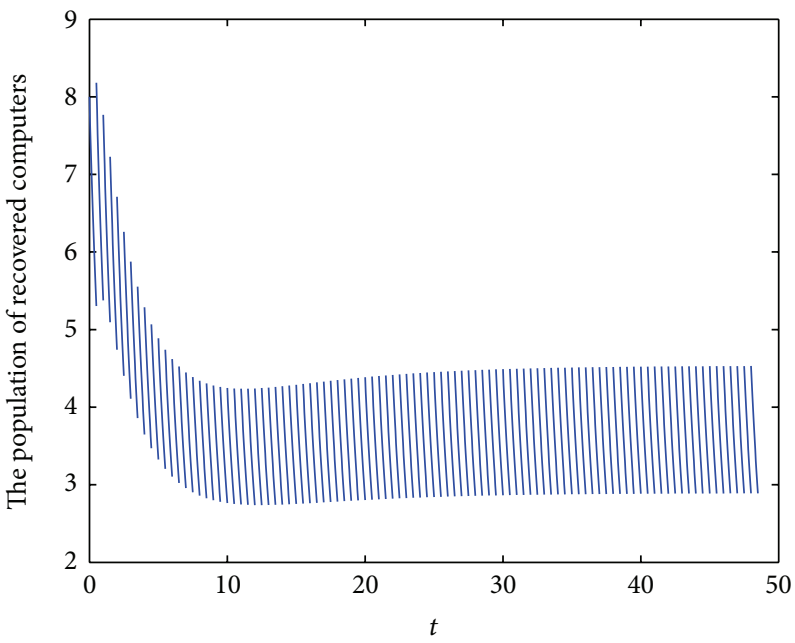

(d)

FIGURE 3: Global attractivity of virus-free periodic solution of system.

From the third equation of (6), we have

$$
\dot{N}(t)>b-\mu N(t)-\alpha N(t)
$$

It is easy to see that

$$
N(t)>\frac{b}{\mu+\alpha}-\varepsilon \doteq m_{N} .
$$

We let $\Omega_{0}=\left\{(S, I, N) \in R_{+}^{3} \mid m_{S} \leq S, m_{I} \leq I, m_{N} \leq N \leq b / \mu\right.$, $S+I \leq b / \mu\}$. By Theorem 6 and the previous discussions, we know that the set $\Omega_{0}$ is a global attractor in $\Omega$, and of course, every solution of system (6) with initial conditions (8) will eventually enter and remain in region $\Omega_{0}$. Therefore, system (6) is permanent.

The proof of Theorem 7 is completed.

Corollary 8. It follows from Theorem 6 that the system (6) is uniformly persistent, provided that $\theta<\theta_{1}$, where $\theta_{1}=1-$ $e^{(\zeta+d) \tau}+\varepsilon b \lambda\left(e^{(\zeta+d) \tau}-1\right) / d[(d+\varepsilon-p b)(r+d+\eta)-\varepsilon q b]$.

\section{Numerical Simulations}

In this section we have performed some numerical simulations to show the geometric impression of our results.

To demonstrate the global attractivity of virus-free periodic solution of system (6), we take following set parameter values: $b=2.1, \lambda=0.2, p=0.1, q=0.15, d=0.3, \zeta=$ $0.6, \varepsilon=0.4, \gamma=0.6, \tau=0.5, \eta=0.3$, and $\theta=0.4$. In this case, we have $R_{0}=0.6886<1$. In Figures 3(a), 3(b), 3(c), and $3(\mathrm{~d})$, we have displayed, respectively, the susceptible, exposed, infected and recovered population of system (6) with initial conditions: $S(0)=6, E(0)=1, I(0)=1$, and $R(0)=8$.

To demonstrate the permanence of system (6), we take the following set parameter values: $b=2.7, \lambda=0.2, p=0.1$, $q=0.15, d=0.3, \zeta=0.6, \varepsilon=0.4, \gamma=0.6, \tau=0.5, \eta=0.3$ and $\theta=0.1$. In this case, we have $R_{1}=1.6496>1$. In Figures $4(\mathrm{a}), 4(\mathrm{~b}), 4(\mathrm{c})$, and 4(d), we have displayed, respectively, the susceptible, exposed, infected, and recovered populations of system (6) with initial conditions: $S(0)=6, E(0)=1, I(0)=1$ and $R(0)=8$. 


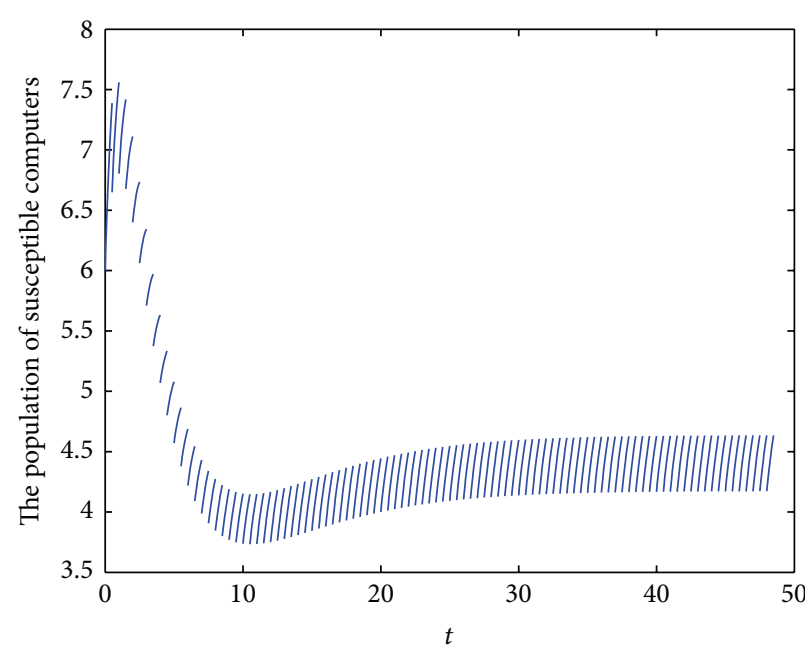

(a)

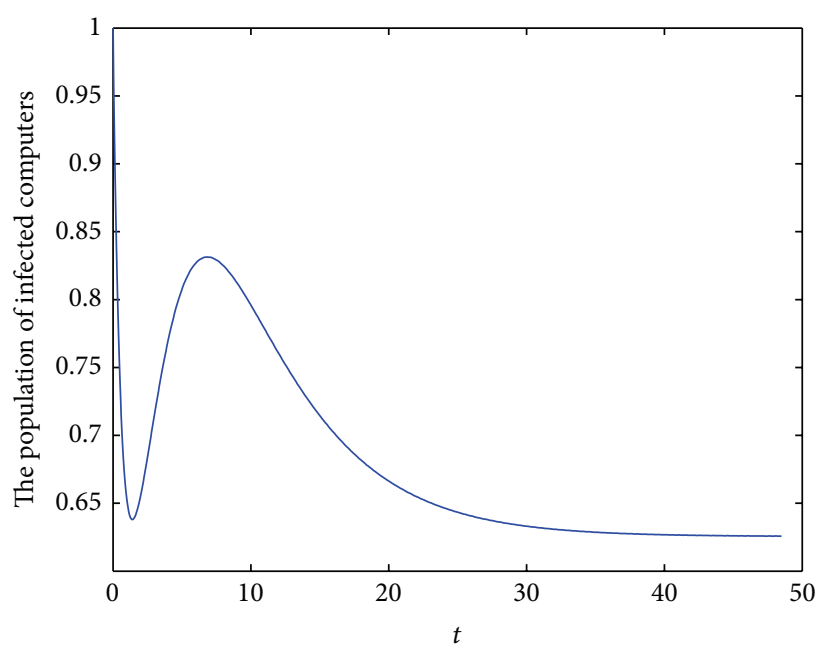

(c)

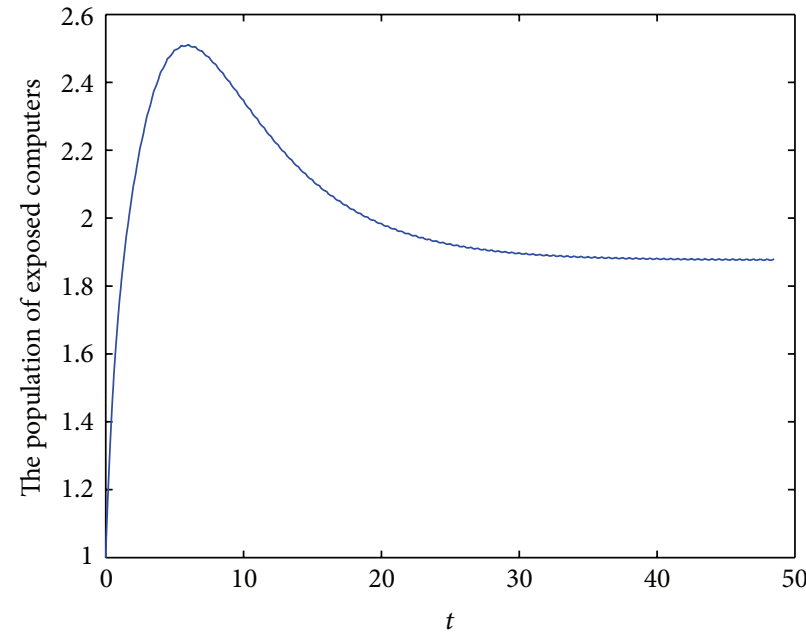

(b)

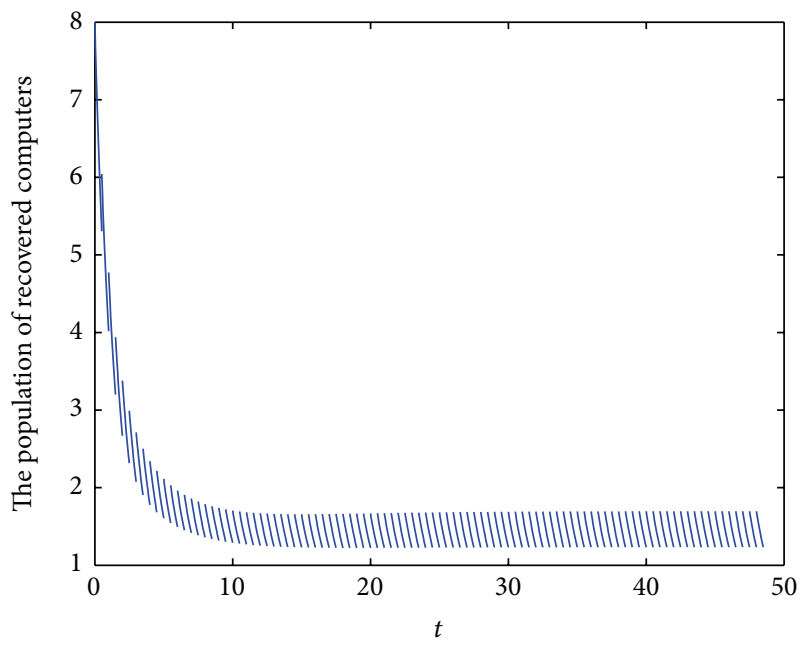

(d)

Figure 4: Permanence of system.

\section{Conclusion}

We have analyzed the SEIRS model with pulse vaccination and varying total population size. We have shown that $R_{1}>1$ or $\theta<\theta_{1}$ implies that the worm will be local, whereas $R_{1}<1$ or $\theta>\theta_{1}$ implies that the worm will fade out. We have also established sufficient condition for the permanence of the model. Our results indicate that a large pulse vaccination rate will lead to eradication of the worm.

\section{References}

[1] J. O. Kephart and S. R. White, "Directed-graph epidemiological models of computer viruses," in Proceedings of IEEE Symposium on Security and Privacy, pp. 343-359, 1991.

[2] J. O. Kephart, S. R. White, and D. M. Chess, "Computers and epidemiology," IEEE Spectrum, vol. 30, no. 5, pp. 20-26, 1993.

[3] L. Billings, W. M. Spears, and I. B. Schwartz, "A unified prediction of computer virus spread in connected networks," Physics Letters A, vol. 297, no. 3-4, pp. 261-266, 2002.
[4] X. Han and Q. Tan, "Dynamical behavior of computer virus on Internet," Applied Mathematics and Computation, vol. 217, no. 6, pp. 2520-2526, 2010.

[5] B. K. Mishra and N. Jha, "Fixed period of temporary immunity after run of anti-malicious software on computer nodes," Applied Mathematics and Computation, vol. 190, no. 2, pp. 12071212, 2007.

[6] B. K. Mishra and D. Saini, "Mathematical models on computer viruses," Applied Mathematics and Computation, vol. 187, no. 2, pp. 929-936, 2007.

[7] B. K. Mishra and S. K. Pandey, "Dynamic model of worms with vertical transmission in computer network," Applied Mathematics and Computation, vol. 217, no. 21, pp. 8438-8446, 2011.

[8] J. R. C. Piqueira and V. O. Araujo, "A modified epidemiological model for computer viruses," Applied Mathematics and Computation, vol. 213, no. 2, pp. 355-360, 2009.

[9] J. R. C. Piqueira, A. A. Vasconcelos, C. E. C. J. Gabriel, and V. O. Araujo, "Dynamic models for computer viruses," Computers \& Security, vol. 27, no. 7-8, pp. 355-359, 2008. 
[10] J. Ren, X. Yang, L.-X. Yang, Y. Xu, and F. Yang, "A delayed computer virus propagation model and its dynamics," Chaos, Solitons \& Fractals, vol. 45, no. 1, pp. 74-79, 2012.

[11] J. Ren, X. Yang, Q. Zhu, L. X. Yang, and C. Zhang, "A novel computer virus model and its dynamics," Nonlinear Analysis, vol. 13, no. 1, pp. 376-384, 2012.

[12] J. C. Wierman and D. J. Marchette, "Modeling computer virus prevalence with a susceptible-infected-susceptible model with reintroduction," Computational Statistics \& Data Analysis, vol. 45, no. 1, pp. 3-23, 2004.

[13] H. Yuan and G. Chen, "Network virus-epidemic model with the point-to-group information propagation," Applied Mathematics and Computation, vol. 206, no. 1, pp. 357-367, 2008.

[14] S. Gao, L. Chen, and Z. Teng, "Impulsive vaccination of an SEIRS model with time delay and varying total population size," Bulletin of Mathematical Biology, vol. 69, no. 1, pp. 731-745, 2007.

[15] R. Shi and L. Chen, "Stage-structured impulsive SI model for pest management," Discrete Dynamics in Nature and Society, Article ID 97608, 11 pages, 2007. 


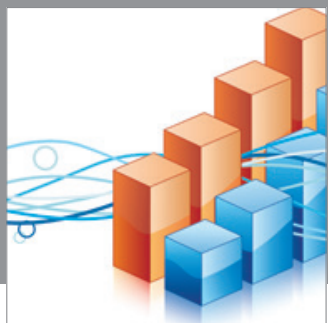

Advances in

Operations Research

mansans

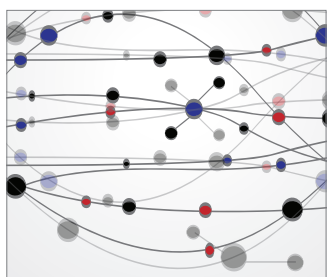

The Scientific World Journal
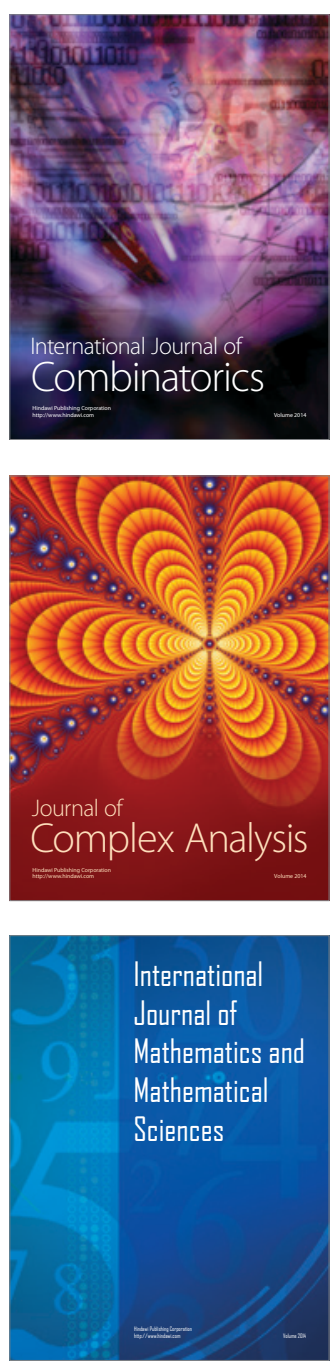
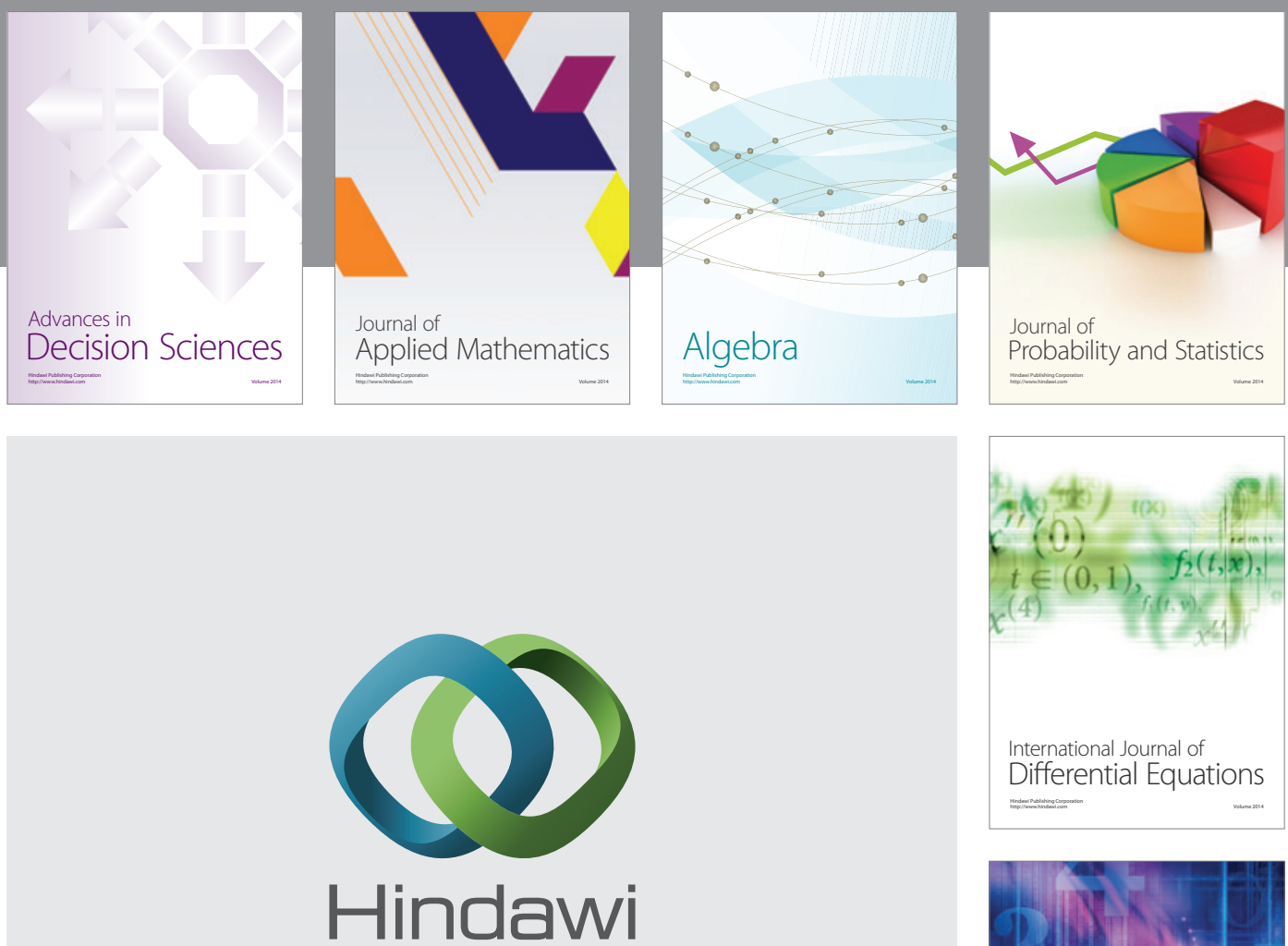

Submit your manuscripts at http://www.hindawi.com
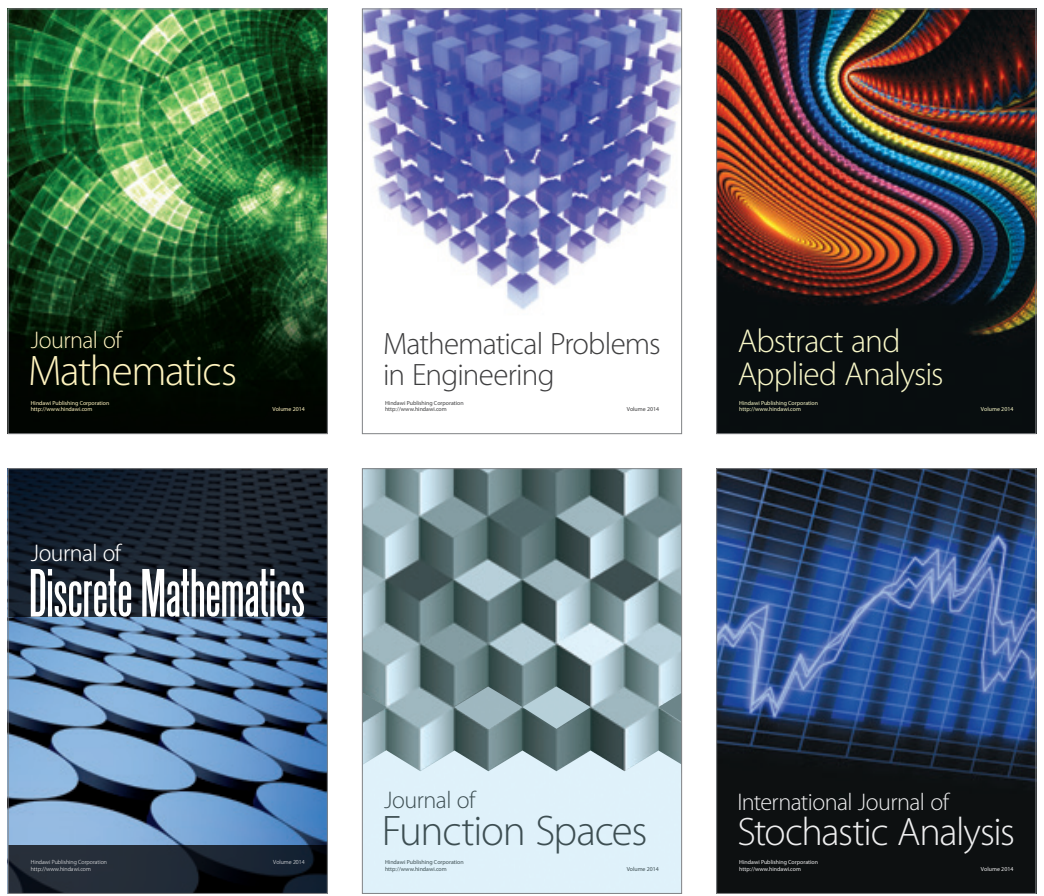

Journal of

Function Spaces

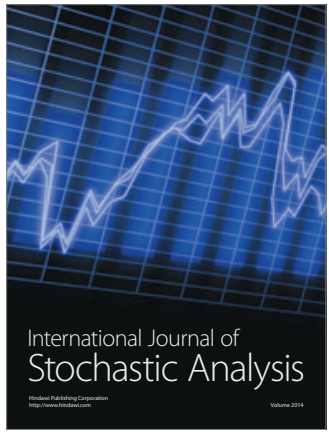

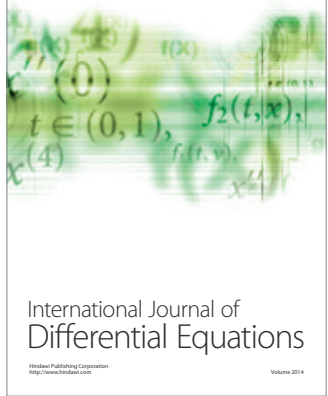
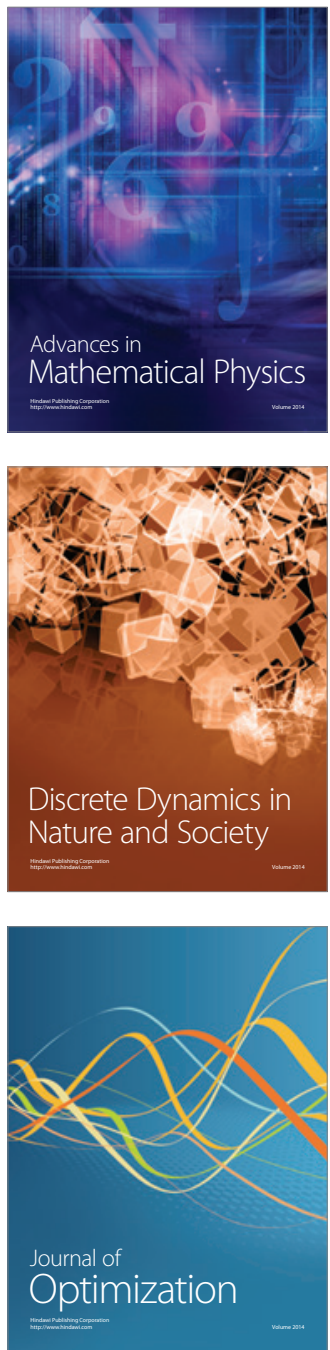